“C 2018 IEEE. Personal use of this material is permitted. Permission from IEEE must be obtained for all other uses, in any current or future media, including reprinting/republishing this material for advertising or promotional purposes, creating new collective works, for resale or redistribution to servers or lists, or reuse of any copyrighted component of this work in other works." 


\section{Perceptual Quality Evaluation of 3D Triangle Mesh: A Technical Review}

\author{
A. A. M Muzahid, Wanggen Wan \\ School of Communications and Information Engineering \\ Institute of Smart City \\ Shanghai University, \\ Shanghai, China \\ muzahid@shu.edu.cn,wanwg@staff.shu.edu.cn
}

\author{
Xiang Feng \\ School of Communications and Information Engineering \\ Institute of Smart City, Shanghai University, Shanghai, China \\ Faculty of Engineering and Information Technology, University of \\ Technology Sydney, \\ Australia \\ fengxiang0727@shu.edu.cn
}

\begin{abstract}
During mesh processing operations (e.g. simplifications, compression, and watermarking), a 3D triangle mesh is subject to various visible distortions on mesh surface which result in a need to estimate visual quality. The necessity of perceptual quality evaluation is already established, as in most cases, human beings are the end users of 3D meshes. To measure such kinds of distortions, the metrics that consider geometric measures integrating human visual system (HVS) is called perceptual quality metrics. In this paper, we direct an expansive study on 3D mesh quality evaluation mostly focusing on recently proposed perceptual based metrics. We limit our study on greyscale static mesh evaluation and attempt to figure out the most workable method for real-time evaluation by making a quantitative comparison. This paper also discusses in detail how to evaluate objective metric's performance with existing subjective databases. In this work, we likewise research the utilization of the psychometric function to expel non-linearity between subjective and objective values. Finally, we draw a comparison among some selected quality metrics and it shows that curvature tensor based quality metrics predicts consistent result in terms of correlation.
\end{abstract}

Keywords-perceptual quality metric; geometric measure; human visual system; curvature tensor; 3D triangular mesh

\section{INTRODUCTION}

With the development in computer graphics and telecommunications, 3D models have been widely used in several application domains including online gaming, computeraided-design (CAD), scientific visualization, medical imaging, digital entertainment, virtual realization, cultural heritage and so on [1]. The 3D model is, in most, represented by triangular mesh that is a discrete representation of geometry including a set of vertices, edges and faces. Utilizing a high number of vertices and faces allow a more detailed information of a mesh that might ensure the eminent visible quality with very high-level details. However, with the objectives of quickening transmission for remote online 3D representation, 3D meshes are subjected to an extensive variety of lossy operations due to rendering or memory utilization and implementation of copyright protection. For example, compression, simplification, filtering and watermarking. These kinds of operations introduce geometric distortions that may alter the visual nature of the $3 \mathrm{D}$ model. To measure the number of distortions is such critical as these processing operations are regularly focused on human-centered applications. Since subjective evaluation (human judgment) is too expensive, laborious and time-consuming, it demands a human perceptual based objective measure model that can meticulously evaluate the distortion taking into account the characteristics of human vision [2]. Up until recently, there are many objective metrics and approaches proposed to evaluate the visual quality of a distorted mesh by computing amount of local geometric measures with HVS function. Simple geometric measures like Hausdorff distance [3] or root mean square error (RMS) [4] generally fail to reflect the perceived visual quality and do not correlate well with human perception. They only consider local geometric distance and make a comparison based on simple similarity measures between the reference mesh and the distorted one. As a matter of fact, there are some specialized components in charge of the geometries of mesh and human visual framework.

In the geometric contortion of mesh, changes in the vertex position and number, vertex removals and rendering algorithm are the most essential key parameters. On the other hand, the perceptibility of surface contortion relies upon a few elements like lighting conditions and directions, viewpoints, viewing distance (long view distance-low perceptibility of distortions) and surface material. During last ten years, several mesh visual quality (MVQ) metrics are introduced [5], mostly work on structural mapping such as Mesh Structural Distortion Measure (MSDM) [6], MSDM2 [7], Dihedral Angle Mesh Error (DAME) [8], Fast Mesh Perceptual Distance (FMPD) [9], Tensor-based Perceptual Distance Measure (TPDM) [10], Dong [11] and TPDMSP [5]. These are the example of objective quality metrics will probably deliver a score that predicts the subjective visual quality regarding a reference model [2]. Target techniques (objective metric) can be categorized depending on the accessibility of the reference: if MVQ metric uses full reference called full-reference model, uses part of reference known as reduced-reference and if there is no reference available called no-reference or blind evaluation [12].

In general, a review paper provides some obvious understanding and key remark on the recent advantages of one area that definitely help the researchers or understudies of that field. Prior to now, there are very few review articles have been published on perceptual quality evaluation. Among of them, some tremendous works are respectively [13], [14], [2] [15]. In fact, to best our knowledge, [2] is the last comprehensive study paper in this field, published in 2013 covering both image and model-based metrics. After that, there are no comprehensive review papers revealed on 3D mesh evaluation, although there are many fruitful approaches and productive methodologies 


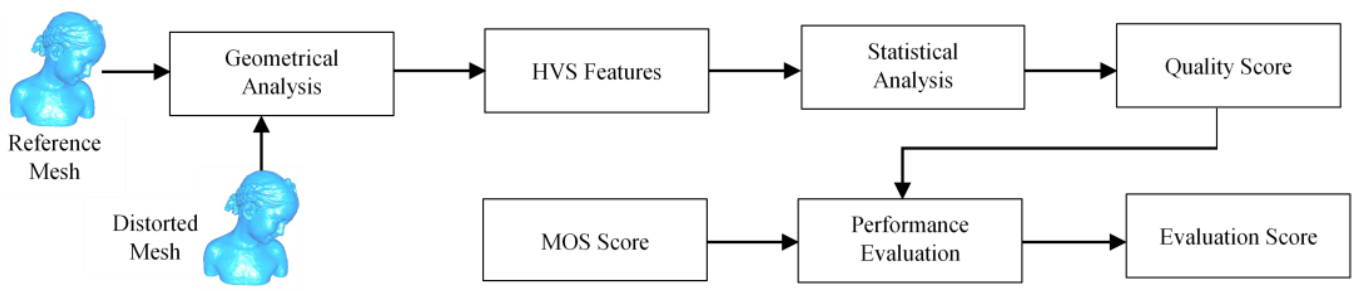

Fig. 1 Overall schematic diagram of 3D mesh visual quality evaluation

published in the following years. Motivating this reality, we intend to write this review paper compiling all the recent findings together and studying with the recent advances and future objectives that might be useful to a new researcher in the field of 3D mesh evaluation. The rest of this paper is organized as follows: section II talks about the basic working principle of visual quality evaluation of $3 \mathrm{D}$ mesh. In section III, a comprehensive study directed on MVQ metrics while implementation and evaluation are briefly discussed in section IV. Furthermore, the experimental results and comparison are reviewed in section V. Finally, we reach the conclusion and future trend in section VI.

\section{Visual Quality Evaluation OF 3D Mesh}

In most cases, the end clients of 3D triangular meshes are human beings, so the application of 3D mesh processing must guarantee high visual quality. Before using mesh processing application, it requires to consider two fundamental information of 3D mesh: 1) possible factors of geometric distortion and 2) human perceptual attributes. These factors are already uncovered by a number of consequence research works. Many of their ideas were taken from image quality evaluation but it is also proved that image-based metric is not able to ensure the perceptual quality of $3 \mathrm{D}$ meshes using $2 \mathrm{D}$ image projections [16]. Subsequently, model-based MVQ metrics are developed even as yet taking a shot at to bring a productive approach that can work in all application domains.

\section{A. Geometric Distortion}

In MVQ evaluation, both mesh surface and geometric structure provide several perceptual features. Variation in a number of vertices alters the vertex connectivity and direction. Indeed, the vertex connectivity also relates to curvature. Conversely, calculation of curvature on each vertex is not such simple with conventional way as a triangle mesh is a piecewise linear surface. Curvature depicts visual attributes (roughness) of 3D surfaces and has been applied in many existing quality assessment strategies [17]. Other mostly used terms of mesh distance calculation are dihedral angle [8], face normal, mean curvature, curvature tensor and vertex sampling distance. The dihedral angle is used to build the concept of global roughness. Mean curvature depicts the deviation measure of a surface of being flat and gives a number of visual attributes of a 3D model, especially sharpness, roughness or smoothness of an area. Among them, curvature tensor is very well known term as it is used in several papers recently [5], [10], [17]. The distance calculation with only geometric quantities just doesn't associate well with the subjective score that requires considering HVS features.

\section{B. Human Perceptual Attributes}

Human vision is exceptionally delicate in light energy, the direction of light, surface smoothness and color contrast [1819]. The viewpoint and distance are also important facts while observing the object. A slightly visible noise may disappear with increasing the distance between object and viewers. Display Size, scene illumination, surface material and rendering algorithm also influence the visibility of geometrical distortion [18]. Taking into account this phenomenon, there are several perceptual properties (Roughness-based, Contrast sensitivity, Visual Saliency, Visual masking and smoothness) weighted with the geometric calculation to get the global perceptual distance of 3D mesh. The more detailed explanation about HVS feature is discussed in the next section.

Fig. 1 depicts the overall workflow of perceptual visual quality evaluation of 3D mesh. The first block is for distance calculation by geometrical analysis e.g. curvature analysis, curvature tensor and so on. Then the geometrical measures are weighted with HVS feature (i.e., saliency mapping). Considering a lot of numerical data, some statistical analysis take part like spatial or Max pooling, Minkowski sum of local distances and many more. After that, the obtained global distance is known as the final quality score. To verify this measure, it requires the performance evaluation with mean opinion score (MOS-subjective score) using correlations. The higher percentage of correlation value refers to strong similarity with the subjective score.

\section{QuALITY METRIC OF PERCEPTUAL EVALUATION}

In general, the quality evaluation of 3D mesh can be divided into two categories: a) image based and b) model based. However, a common question always arises, what type of metrics is perfect to measure 3D mesh visual quality. In [16], they work on this issue and a series of experiments were conducted to address this question. It depicts that, 2Djudgement don't give a decent indicator of 3D mesh quality evaluation, it requires object quality metrics. In the following, [20] conducts another experiment and reveals some contradictory comments though they just consider a single type distortion (simplification). There was no clear explanation for other types of distortions. Two years ago, a new and fruitful experimental review published [15]. All experiments and discussions are nicely placed. It concludes the image-based metric might be a great indicator relying upon the nature of

artifacts. In simple and rendering scenario under a single type of distortion, image-based metrics perform very well regards to model-based metrics. In addition, as of late, the model-based metrics provide significantly better results for extreme 

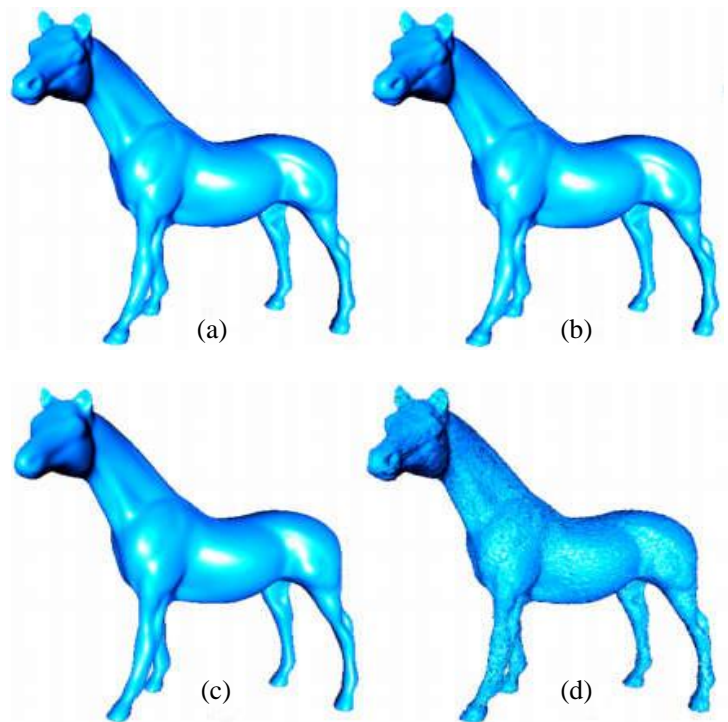

Fig. 2 Original and distorted versions of the Horse model, all associated with the same maximum root mean square error $\left(1.05 \times 10^{-3}\right)$. (a) Original model. (b) watermarked model [21] (c) Laplacian smoothing [22] and (d) Gaussian noise addition from. From [11]

scenarios. For instance, evaluating various types of distortions, model-based metrics are very accurate at differentiating the artifacts between different distortions. In [14], authors discussed quality metrics based on viewing conditions categorized by viewpoint-independent and viewpointdependent quality assessment.

Apart from them, a comprehensive study on quality metrics is considered in this review rather separating them in a class.

A. Geometric-distance-based:

Root mean square (RMS) is the earliest example that belongs to this family. It's a full reference technique that calculates the similarity (point to point) between two meshes. The equation can be written as follows:

$$
\operatorname{RMS}(A, B)={\sqrt{\sum_{j=1}^{n}\left\|a_{j}-b_{j}\right\|^{2}}}^{2},
$$

$\mathrm{A}$ and $\mathrm{B}$ are two meshes that carry the same number of vertices where $a_{j}$ and $b_{j}$ are the corresponding points on vertices. The Euclidean distance between two points refers to as $\|.$.$\| . The$ issue is that this metric is restricted to comparing meshes with a similar number of vertices and connectivity [14]. This connectivity issue was solved further by Hausdorff distance [13]. The distance from a point $p$ in the 3D space and 3D object defines by $e(p, A)$ :

$$
e(p, A)=\min _{v_{A}^{i} \in A} d\left(p, v_{A}^{i}\right)
$$

where $d=$ the Euclidian $v_{A}^{i}=i^{t h}$ vertex of object $A$. Then the asymmetric and symmetric Hausdorff distance between two 3D objects A and B is represented by (2) and (3) respectively.

$$
\begin{gathered}
H_{a}(A, B)=\max _{v_{A}^{i} \in A} e\left(v_{i}^{A}, B\right), \\
H_{d}(A, B)=\max \left\{H_{a}(A, B), H_{a}(B, A)\right\} .
\end{gathered}
$$

However, both RMS and Hausdorff distance metrics fail to correlate with human vision. Fig. 2 shows several 3D meshes with same RMS value, but in reality, each of them has a different visual appearance.

\section{B. Curvature-based}

The curvature changes of triangular mesh affect the human eyesight and a discrete differential error metric (DDEM) was proposed in [23] for surface simplification. Thereafter, there were some more fruitful works proposed on the basis of it. [6] proposed mesh structural distortion measure (MSDM) using the concept of structural similarity based on curvature analysis (mean, standard deviation and covariance) on the local window. It was demanded, this tool could be used for any kinds of 3D mesh processing algorithms. This work follows the idea of 2Dimage quality evaluation using structural similarity measures by [24]. MSDM is written as follows:

$$
\operatorname{LMSDM}(x, y)=\left(\alpha \times L(x, y)^{a}+\beta \times C(x, y)^{a}+\gamma \times S(x, y)^{a}\right)^{\frac{1}{a}}
$$

where $L=$ curvature distance lead rendering \& visual appearance. $C=$ contrast comparison based on the standard deviation that reflects the roughness of the surface. $S=$ detect the changes of salient features measuring covariance between the local windows. Static parameters $a, \alpha, \beta$ and $\gamma$ are chosen respectively to $3,0.4,0.4$, and 0.2 . The global distance is measured by (6),

$$
\operatorname{MSDM}(X, Y)=\left(\frac{1}{M} \sum_{j=1}^{M} \operatorname{LMSDM}\left(x_{j}, y_{j}\right)^{a}\right)^{\frac{1}{a}}
$$

here $M$ is the number of the local windows, $x_{j}$ and $y_{j}$ are the local contents of the local window. The value of MSDM lies between 0 to 1 . Multi-resolution constraint-free new metric MSDM2 proposed in [7] with improved quality that relates a vertex matching preprocessing with different vertex connections. MSDM2 reveals a better correlation with the subjective score. Tensor-based perceptual distance metric, TPDM, was proposed by [17] taking into account both curvature amplitude and direction. It also accounts for visual masking effect through a roughness-based weighting of the local tensor. The global measures of TPDM is calculated as:

$$
T P D M=\left(\sum_{i=1}^{N} w_{i}\left|\operatorname{LTPDM}_{v_{i}}\right|^{p}\right) \frac{1}{p},
$$

where $L T P D M_{v_{i}}$ represents the local tensor distance between the vertices $v_{i}(i=1,2, \ldots ., N)$ of the reference and the distorted mesh and $w_{i}$ is the area of weighting coefficients. For more details on TPDM, readers can refer to [10], [17]. Another curvature based perceptual quality metric is proposed in [25]. It computes mean curvature and this computation results are adjusted further with a visual masking and saturation components. Quality score is determined from a mixture of the mean curvature variation and distortions of the structure. There are also few more approaches proposed based on curvature and curvature tensor information respectively [11] and [26-27].

\section{Dihedral Angle}

Dihedral angle differences is another approach to $3 \mathrm{~d}$ mesh evaluation as it takes into account face normalization and vertex 
differences between reference and distorted mesh. [8] proposed a metric based on local changes of oriented dihedral angles, since it's was linked to quadratic bending energy function [28]. Let $t_{1}$ and $t_{2}$ are two adjacent triangles and each of them contains vertices respectively $v_{1}, v_{2}$ and $v_{3}$ with $n_{1}$ and $n_{2}$ faces (normalized). According to [8], dihedral angle is calculated as follows:

$$
D_{t_{1}}, t_{2}=\arccos \left(n_{1} \cdot n_{2}\right) * \operatorname{sgn}\left(n_{1} \cdot\left(v_{4}-v_{3}\right)\right), \quad \in[-\pi, \pi]
$$

If two triangles lie on the same plane then the value of $D_{t_{1}}, t_{2}$ is equal to zero (0). The possible viewing direction was also taken into account and weighted by detecting smoothness measure with low magnitude dihedral angles. The final measures of dihedral angle mesh error (DAME) are obtained by summing up contributions from all edges. These measures limit to same connectivity only.

Similar to [8], a new MVQ metric proposed in [29] based on also dihedral angles using statistical distributors to extract the parameters. It also considers masking effect based on roughness rather than smoothness by selecting high magnitudes dihedral angles. The final objective score is calculated by Kullback-Leibler divergence between original and distorted distributions. Of later, no reference perceptual metric proposed in [30] using dihedral angles where support vector regression (SVR) is used to generate the objective score. It's likewise associated with visual masking effect by deploying roughness model. Dihedral angles provide as structural information of mesh then it's weighted with visual masking features. Finally, Gamma distribution is utilized to extract the feature to connect with SVR in order to get an objective score.

\section{Machine Learning}

The application of Machine learning (ML) is widely used in the domain of computer graphics. In [31], an ML used to validate hypothesis further fitted to get a quality score of 3D mesh visual assessment. Authors proposed a new approach to quality metric using machine learning strategies based on an optimized combination of geometric attributes. All parameters of a multiple feature metric are upgraded utilizing machine learning techniques. They directed a series of experiments using 8 -conventional metrics. It uncovers that the attributes of curvature analysis (Mean \& Max) and dihedral angles provide better correlation with subjective scores. From this motivation, they assume (based on hypothesis) that a mixture of these attributes ought to significantly enhance the prediction performance. To approve this hypothesis, the quality predictor $P_{i}$ is ascertained using a linear combination of the 8 attributes (total number conventional metrics used in the experiment) is as follows:

$$
P_{i}=\omega_{0}+\sum_{i=1}^{8} \omega_{i} \cdot \Delta a_{i}
$$

where $\Delta a_{i}$ is the attribute metric ( $i=1$ to 8 ) and $\omega_{i}$ are the weights. Multi-linear regression model based on the Akaike information criterion [32] is used to train the weights $\omega_{i}$. The weight was trained on a random subset of half of the models and tested on the other half. The predicted objective score was considerably improved the prediction efficiency. The total systems comparatively simple to implement and also requires lower execution time. Two years' prior, [5] proposed another perceptual quality metric using the machine learning paradigm. It deploys TPDM [10] metric to give perceptual distortion based on carver tensor analysis. They likewise proposed to use statistical descriptor (spatial pooling) to extract feature vector extraction. At long last, SVR (support vector) employed as the learning model to learn the nonlinear complex relationship between feature vector and the MOS score. This newly designed objective metric meet acceptable performance even with a small amount of training dataset. There are few more objective metrics based on ML will be discussed in the following section.

\section{E. Blind Evaluation}

Among of three type of mesh evaluation, no reference evaluation known as blind evaluation. The big challenge of this type of evaluation is to automatically predict the perceived quality when the reference is unavailable. One of the earliest work on blind evaluation proposed in [30], that already discussed in subsection $C$. In addition, the final objective measure of this metric was compared with full-reference and reduced-reference objective scores. However, a prominent result achieved by no-reference based-model.

In [26], authors proposed curvature based blind 3D mesh evaluation using general regression neural network (GRNN). This GRNN also can work with relatively smaller data set during a training session. A new approach based on multi-scale saliency and roughness maps called Blind Mesh Quality Index (MBQI) metric proposed in [33]. It also uses SVR for feature vector extraction with a specific end goal to get last target quality score. A deep learning approach was proposed by [34] employing multilayer $\mathrm{CNN}$ with multilayer max-pooling. Similar to previous, it also considers curvature and dihedral angles from each distorted mesh. As of late, [12] proposed with a similar approach of blind 3D visual quality assessment using SVR with an improved result. In the training session, 3 openly accessible databases are used, perhaps, the reference mesh of target-distorted one never undergoes into the training session. This phenomenon clearly make sure about its no reference evaluation. However, this proposed model use three set of feature vector using tree statistical destitutions: Gamma, Weibull and Rayleigh. A weighted sum of three intermediate scores from statistical distortion led to getting a final score of the objective metric.

\section{F. Others}

Due to space limitation, we got to limit our study, however, numerous popular methodologies are also used for 3D mesh evaluation such as roughness based, visual discrimination model, strain energy based, texture geometry, features fusion and so on. [35] Recently, some productive and new findings have been revealed on special characteristics of the 3D mesh such as visual attention for rendered 3D shape [18], [19], [36], just noticeable distortion profile and visual contrast sensitivity. These kinds of new findings can be used further to develop a new perceptual model for 3D mesh evaluation.

\section{IMPLEMENTATION AND EVALUATION}

Implementation and evaluation, these two are the most cruc- 
TABLE I. A quantitative comparison among correlation coefficients of different objective metrics

\begin{tabular}{|l|c|c|c|c|c|c|c|c|}
\hline \multirow{2}{*}{$\begin{array}{l}\text { Objective } \\
\text { Metrics }\end{array}$} & \multicolumn{2}{|c|}{$\begin{array}{c}\text { General-purpose } \\
\text { Database }\end{array}$} & \multicolumn{2}{c|}{$\begin{array}{c}\text { Simplification } \\
\text { Database }\end{array}$} & \multicolumn{2}{c|}{$\begin{array}{c}\text { Masking } \\
\text { Database }\end{array}$} & \multicolumn{2}{c|}{$\begin{array}{c}\text { Compression } \\
\text { Database }\end{array}$} \\
\cline { 2 - 9 } & PLCC & SROCC & PLCC & SROCC & PLCC & SROCC & PLCC & SROCC \\
\hline HD [3] & 11.4 & 13.8 & 50.5 & 49.4 & 20.2 & 26.6 & 14.0 & 24.5 \\
\hline RMS [4] & 28.1 & 26.8 & 59.6 & 70.2 & 41 a2 & 48.8 & 49 & $5(\mathbf{B})$ \\
\hline MSDM [6] & 75.0 & 73.9 & N/A & N/A & 69.2 & 65.2 & 91.5 & 83.1 \\
\hline MSDM2 [7] & 81.4 & 80.4 & 89.2 & 86.7 & 87.3 & 89.6 & 89.3 & 78.0 \\
\hline TPDM [10] & 86.2 & 89.6 & $\mathbf{8 6 . 9}$ & $\mathbf{8 8 . 2}$ & 88.6 & 90.0 & 91.5 & 82.9 \\
\hline VMSC [14] & 87.7 & 86.6 & N/A & N/A & $\mathbf{9 2 . 6}$ & $\mathbf{9 2 . 5}$ & $\mathbf{9 6 . 3}$ & 84.2 \\
\hline TPDMSP [5] & $\mathbf{9 5 . 9}$ & $\mathbf{9 6 . 1}$ & N/A & N/A & NA & NA & 91.5 & 83.5 \\
\hline Blind-1 [34] & 82.7 & 83.6 & N/A & N/A & 85.4 & 88.2 & NA & NA \\
\hline Blind-2 [12] & 86.8 & 84.6 & N/A & N/A & 89.1 & 91.1 & 88.1 & $\mathbf{8 5 . 5}$ \\
\hline
\end{tabular}

ial parts of $3 \mathrm{~d}$ mesh evaluation due to the complex nature of $3 \mathrm{~d}$ triangular mesh. after getting the distance score from objective metric, it's expected to approve this measure and observed, how does it associate with human eyes. to lead this sort of test, we must need some subjective mos databases, where several types of the distorted samples of $3 \mathrm{~d}$ meshes will be accessible with their corresponded moss finally, we have to direct some particular operation like the cross-correlation between subjective and objective scores.

\section{A. Subjective Score and Databases}

It's another critical part to make subjective scores. In early works [37]-[39], they conducted series of subjective measures by the direct observers. In that case, the original and several distorted (noise addition, compressed and simplified) models from the corpus are displayed to the observers. The subjects were asked to give score reflecting the degree of perceived distortion in the range from 0 to 10 (identical to worst case). However, the scoring system can be categorized with adjectival (bad, poor, fair, good, excellent), numerical $(1,2,3,4,5)$ or on a continuous scale $(\in[0,100])$. The MOS can be computed individually for each distorted model of the corpus [13]:

$$
\operatorname{MOS}_{i}=\frac{1}{n} \sum_{j=1}^{n} m_{i j} .
$$

where $n$ is the total number of the test object, $m_{i j}$ is the score given by $j^{\text {th }}$ object to the $i^{\text {th }}$ object and $M O S_{i}$ is the final mean opinion score of the $i^{\text {th }}$ object. Perhaps, there are a couple of methods with a complete set of rules are well described in [2]. Finally, the MOS values are normalized to avoid some misuses of a possible outlier. To assume the grading scale and MOS score, different methodologies are used for different purposes [14]. For example, double-stimulus continuous qualityscale(DSCQS) for a system against a reference and simultaneous double stimulus for continuous evaluation (SDSCE) for a video sequence. However, there are some special parameters i.e. lighting condition, direction of light, background, material properties (e.g. texture and normal) and shading, animation and interaction, types of objects, masking effect, levels, stimuli order and display duration taken into account during the subjective evaluation as the visual quality may affect by them [14]. These types of artifacts are already
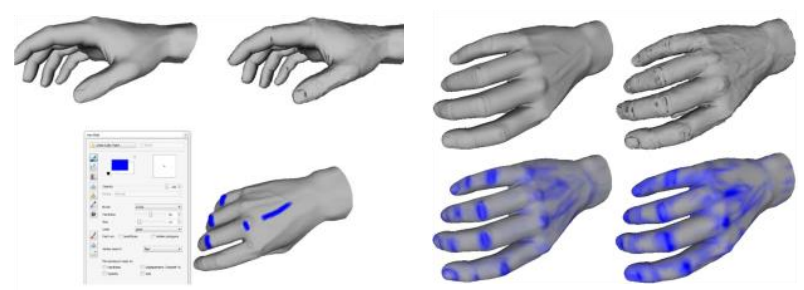

Fig. 3 Experimental set up for hand mash distortion identification. a) Evaluation Screen b) Top-left: reference mesh, top-right: modified mesh, bottom-left: mean subjective response, bottom-right: estimated visual response. From [40]

proved and experimented by several researchers which are already discussed in the previous section. In [40], a marking tool and marking system of subjective responses on distorted areas are described clearly, shown in Fig. 3. The list of some existing greyscale 3D mesh databases are listed below:

1. LIRIS/EPFL General-purpose database [6]

2. LIRIS Masking database [41]

3. IEETA Simplification database [42]

4. UWB compression database [8]

5. LIRIS Localized Geometric Artefacts Database [27]

To our knowledge, there is no colored 3D mesh subjective database proposed yet, but recently [43] build a database of the colored 3D model without MOSs. It contains 425 coloredmodels were acquired from 15 real objects. Several distortions have been applied on rough or smooth areas according to different strengths and situations

\section{EXPERIMENTAL RESULTS}

The objective score of the distorted mesh is normally given by the quality metric. The reliability or the performance of the MVQ metrics are measured with an extensive quantitative comparison of existing MOS databases. In general, two types of correlation are considered: the Spearman rank-order correlation coefficient (SROCC) and the Pearson linear correlation coefficient (PLCC) [5]. The SROCC measures the monotonic association between the two ranked variables. On the other hand, PLCC measures the prediction accuracy of the objectives metrics where a non-linear regression (Gaussian 


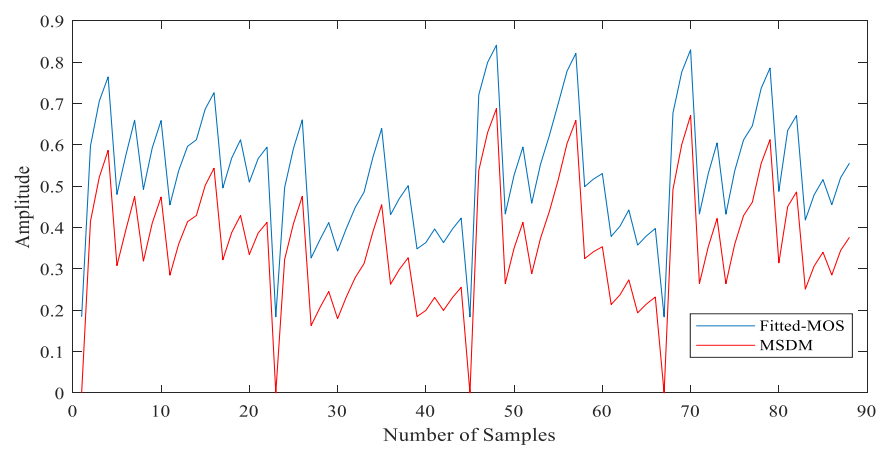

Fig. 4 Comparison between fitted MOS and MSDM values

psychometric function) on the metric values are conducted prior to PLCC calculation [44]. The PLCC and SROCC between two variables $x$ and $y$ are calculated as follows:

$$
\begin{gathered}
P L C C=\frac{\sum_{i=1}^{n}(x-\bar{x})(y-\bar{y})}{\sqrt{\sum_{i=1}^{n}\left(x_{i}-\bar{x}\right)^{2}} \sqrt{\sum_{i=1}^{n}(y-\bar{y})^{2}}} \\
\text { SROCC }=1-\frac{6 \sum_{i=1}^{N}\left(X_{i}-Y_{i}\right)^{2}}{n\left(n^{2}-1\right)}
\end{gathered}
$$

To remove the non-linearity between subjective and objective scores, a cumulative Gaussian psychometric function is calculated prior to PLCC can be written as follows [44]:

$$
g(a, b, S)=\frac{1}{\sqrt{2 \pi}} \int_{a+b S}^{\infty} e^{\left(-t^{2} / 2\right)} d t
$$

where $S$ is the objective metric values. Parameters $a \& b$ is obtained through a non-linear least squares fitting using MATLAB. However, this psychometric function never changes the SROCC value. To verify this characteristic, we conduct an experiment for MSDM [6] objective metric on LIRIS/EPFL General-purpose database. Due to different measurement scale, at first, we convert MOSs and MSDM values to the same scale between 0 to 1 and then fed to curve fitting block. Fig. 4. depicts a comparison between fitted MOSs and MSDM values. Finally, the PLCC is calculated between fitted MOSs and Original MOSs values. The obtained PLCC after the fitting is improved from 74.1 to 75.1 but SROCC is remained same to 73.91. The correlation results are perfectly similar to the revised metrics comparison Table 3 in [2]. Table I depicts a comparison of different quality metrics from prior to most recent ones. All quantitative scores are collected from respected paper; no value has been reproduced. It can be seen that there is no single metrics that can work better for all kinds of distortions. Most recent Blind-2 [12] works better in compression database in terms of SROCC (85.5) but it fails to correlate on other databases. TPDMSP [5] provides a promising score than all state-of-art metrics on the general-purpose database. However, in order to fit of real-time applications, we need to emphasize more on blind evaluation metric.

\section{CONCLUSION AND FUTURE TREND}

The recent perceptual metrics for 3D mesh evaluation are explored in this paper. We led both qualitative and quantitative study and also drew a comparison between the performance of

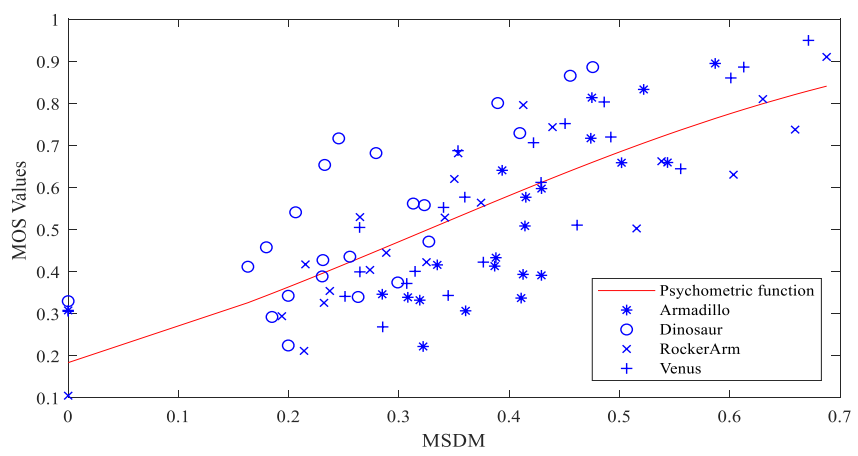

Fig. 5 Psychometric function curve plotted with MSDM-MOS pairs on LIRIS/EPFL general-purpose database

today's evaluation metrics and those of a decade ago to figure out the challenges and advances of them. Although some perceptually based metrics made some great progress but evaluating the perceptibility of distortion is still an open issue with many unresolved issues. Recently, machine learning approach was introduced with great precision and high correlation between the subjective and objective scores, however, the lack of training 3D mesh models is one of the typical barriers to implementing in real-time. Another practical approach is blind mesh evaluation that is still in early stage but could be the conceivable fruitful approach in the real-time evaluation. From the Table I, we couldn't find any ideal metric that can be used to detect any kinds of distortions. In some specific areas, individual perceptual metrics performed sound great like TPDM, TPDMSP, VMSC and Blind-2 on Simplification, General-purpose and Compression databases respectively.

Nowadays, colored 3D models are widely used in almost every field of computer graphics, but as far as we know, there is no metric has been introduced yet for $3 \mathrm{D}$ colored mesh evaluation. Even there is no subjective database of colored mesh proposed yet. In the future, the possible research direction of us would be to develop a subjective database for colored mesh and then to investigate some metrics compatible with colored 3D mesh evaluation.

\section{ACKNOWLEDGMENT}

This work is supported by the National Natural Science Foundation of China (No. 61711530245) and the Key Project of Shanghai Science and Technology Commission (No.17511106802).

\section{REFERENCES}

[1] M. Botsch, L. Kobbelt, M. Pauly, P. Alliez, and B. Levy, Polygon Mesh Processing. A K Peters/CRC Press, 2010.

[2] M. Corsini, M. C. Larabi, G. Lavoué, O. Petřík, L. Váša, and K. Wang, "Perceptual Metrics for Static and Dynamic Triangle Meshes," Comput. Graph. Forum, vol. 32, no. 1, pp. 101-125, Feb. 2013.

[3] N. Aspert, D. Santa-Cruz, and T. Ebrahimi, "MESH: Measuring Errors Between Surfaces Using the Hausdorff distance," IEEE International Conference on Multimedia and Expo, vol. 1, pp. 705-708, 2002,

[4] P. Cignoni, C. Rocchini, and R. Scopigno, "Metro: Measuring Error on Simplified Surfaces," Comput. Graph. Forum, vol. 17, pp. 167-174, Jun. 1998. 
[5] X. Feng, W. Wan, R. Y. D. Xu, H. Chen, P. Li, and J. A. Sánchez, "A perceptual quality metric for 3D triangle meshes based on spatial pooling," Front. of Computer Science, pp.1-15, Dec. 2017.

[6] G. Lavoué, E. Drelie Gelasca, F. Dupont, A. Baskurt, and T. Ebrahimi, "Perceptually driven 3D distance metrics with application to watermarking," SPIE Proceedings, vol. 6312, Aug. 2006.

[7] Guillaume Lavoué. A Multiscale Metric for 3D Mesh Visual Quality Assessment. Computer Graphics Forum, Wiley, pp.1427-1437, 2011.

[8] Váša Libor and Rus Jan, "Dihedral Angle Mesh Error: a fast perception correlated distortion measure for fixed connectivity triangle meshes," Comput. Graph. Forum, vol. 31, pp. 1715-1724, Aug. 2012.

[9] K. Wang, F. Torkhani, and A. Montanvert, "A fast roughness-based approach to the assessment of 3D mesh visual quality," Comput. Graph., vol. 36, pp. 808-818, Nov. 2012.

[10] F. Torkhani, K. Wang, and J.-M. Chassery, "A Curvature-Tensor-Based Perceptual Quality Metric for 3D Triangular Meshes," Machine Graphics \& Vision, pp.1-25, 2014,

[11] L. Dong, Y. Fang, W. Lin, and H. S. Seah, "Perceptual Quality Assessment for 3D Triangle Mesh Based on Curvature," IEEE Trans. Multimed., vol. 17, pp. 2174-2184, Dec. 2015.

[12] I. Abouelaziz, M. El Hassouni, and H. Cherifi, "Blind 3D mesh visual quality assessment using support vector regression," Multimed. Tools Appl., Feb. 2018.

[13] G. Lavoue and M. Corsini, "A Comparison of Perceptually-Based Metrics for Objective Evaluation of Geometry Processing," IEEE Trans. Multimed., vol. 12, pp. 636-649, Nov. 2010.

[14] A. Bulbul, T. Capin, G. Lavoue, and M. Preda, "Assessing Visual Quality of 3-D Polygonal Models,” IEEE Signal Process. Mag., vol. 28, pp. 8090, Nov. 2011.

[15] G. Lavoue, M. C. Larabi, and L. Vasa, "On the Efficiency of Image Metrics for Evaluating the Visual Quality of 3D Models," IEEE Trans. Vis. Comput. Graph., vol. 22, pp. 1987-1999, Aug. 2016.

[16] B. E. Rogowitz and H. E. Rushmeier, "Are image quality metrics adequate to evaluate the quality of geometric objects?" in Human Vision and Electronic Imaging VI, vol. 4299, pp. 340-349, 2001.

[17] F. Torkhani, K. Wang, and J.-M. Chassery, "A Curvature Tensor Distance for Mesh Visual Quality Assessment," in Computer Vision and Graphics, vol. 7594, pp. 253-263, 2012

[18] G. Nader, K. Wang, F. Hetroy-Wheeler, and F. Dupont, "Just Noticeable Distortion Profile for Flat-Shaded 3D Mesh Surfaces," IEEE Trans. Vis. Comput. Graph., vol. 22, pp. 2423-2436, Nov. 2016.

[19] G. Lavoué, F. Cordier, H. Seo, and M. C. Larabi, "Visual Attention for Rendered 3D Shapes," Euro graphics, vol. 37, 2018.

[20] I. Cleju and D. Saupe, "Evaluation of supra-threshold perceptual metrics for 3D models," APGV '06 Proceedings of the 3rd symposium on Applied perception in graphics and visualization, pp. 41-44, 2006.

[21] K. Wang, G. Lavoué, F. Denis, and A. Baskurt, "Robust and blind mesh watermarking based on volume moments," Comput. Graph., vol. 35, pp. 1-19, Jan. 2011.

[22] G. Taubin, "Geometric Signal Processing on Polygonal Meshes," Euro graphics 2000.

[23] S.-J. Kim, S.-K. Kim, and C.-H. Kim, "Discrete differential error metric for surface simplification," $10^{\text {th }}$ Pacific Conference on Computer Graphics and Applications, pp. 276-283, 2002.

[24] Z. Wang, A. C. Bovik, H. R. Sheikh, and E. P. Simoncelli, "Image quality assessment: from error visibility to structural similarity," IEEE Trans Image Process., vol. 13, pp. 600-612, Apr. 2004.

[25] Lu Dong, Yuming Fang, Weisi Lin, and Hock Soon Seah, "Objective visual quality assessment for 3D meshes," pp. 1-6, 2014
[26] I. Abouelaziz, M. El Hassouni, and H. Cherifi, "A Curvature Based Method for Blind Mesh Visual Quality Assessment Using a Genera Regression Neural Network," $12^{\text {th }}$ International Conference on SignalImage Technology \& Internet-Based Systems pp. 793-797, 2016.

[27] J. Guo, V. Vidal, A. Baskurt, and G. Lavoué, "Evaluating the local visibility of geometric artifacts," pp. 91-98, 2015.

[28] WARDETZKY M., BERGOU M., HARMON D., ZORIN D., GRINSPUN E.: Discrete quadratic curvature energies. Comput. Aided Geom. Des. 24, pp. 499-518, 2007.

[29] I. Abouelaziz, M. Omari, M. E. Hassouni, and H. Cherifi, "Reduced Reference 3D Mesh Quality Assessment Based on Statistical Models," in 2015 11th International Conference on Signal-Image Technology Internet-Based Systems (SITIS), pp. 170-177, 2015.

[30] I. Abouelaziz, M. E. Hassouni, and H. Cherifi, "No-Reference 3D Mesh Quality Assessment Based on Dihedral Angles Model and Support Vector Regression,” Image and Signal Processing, pp. 369-377, 2016.

[31] G. Lavoue, I. Cheng, and A. Basu, "Perceptual Quality Metrics for 3D Meshes: Towards an Optimal Multi-attribute Computational Model,' IEEE International Conference on Systems, Man, and Cybernetics, Manchester, pp. 3271-3276, 2013.

[32] H. Akaike, "Information Theory and an Extension of the Maximum Likelihood Principle," Selected Papers of Hirotugu Akaike, Springe in Statistics, pp. 199-213, 1998,

[33] A. Nouri, C. Charrier, and O. Lézoray, "3D Blind Mesh Quality Assessment Index,” Electron. Imaging, vol. 20, pp. 9-26, Jan. 2017.

[34] I. Abouelaziz, M. E. Hassouni, and H. Cherifi, "A convolutional neura network framework for blind mesh visual quality assessment," IEEE International Conference on Image Processing, pp. 755-759, 2017.

[35] A. Chetouani, "A 3D Mesh Quality Metric based on Features Fusion," Electron. Imaging, vol. 20, pp. 4-8, Jan. 2017

[36] G. Nader, K. Wang, F. Hétroy-Wheeler, and F. Dupont, "Visual Contras Sensitivity and Discrimination for 3D Meshes and their Applications,' Comput. Graph. Forum, vol. 35, pp. 497-506, Oct. 2016.

[37] S. Silva, C. Ferreira, J. Madeira, and B. S. Santos, "Perceived Quality of Simplified Polygonal Meshes: Evaluation using Observer Studies," Human Vision and Electronic Imaging, vol. 6806, 2008.

[38] B. S. Santos, S. Silva, C. Ferreira and J. Madeira, "Comparison of methods for the simplification of mesh models of the lungs using quality indices and an observer study," Third International Conference on Medical Information Visualisation--BioMedical Visualisation, pp. 15-21 2005.

[39] S. Silva, B. Sousa Santos, J. Madeira, and C. Ferreira, "Perceived quality assessment of polygonal meshes using observer studies: a new extended protocol," Proc. SPIE 6806, Human Vision and Electronic Imaging III, 68060D, 2008.

[40] Z. C. Yildiz and T. Capin, "A perceptual quality metric for dynamic triangle meshes," EURASIP J. Image Video Process., vol. 1, Dec. 2017.

[41] G. Lavoué, "A local roughness measure for 3D meshes and its application to visual masking," ACM Trans. Appl. Percept., vol. 5, pp. 1-23, Jan. 2009 .

[42] S. Silva, B. S. Santos, C. Ferreira, and J. Madeira, "A Perceptual Data Repository for Polygonal Meshes," in 2009 Second International Conference in Visualisation, pp. 207-212, 2009

[43] A. Nouri, C. Charrier, and O. Lézoray, "Technical report: Greyc 3D colored mesh database," Normandie Université, Unicaen, EnsiCaen, CNRS, GREYC UMR 6072, 2017.

[44] X. Feng, W. Wan, R. Y. D. Xu, P. Stuart, L. Pengfei, Z. Song,"A novel spatial pooling method for 3D mesh quality assessment based on percentile weighting strategy," Computers \& Graphics, pp. 1-11, 2018. ( doi.org/10.1016/j.cag.2018.04.005). 